\title{
Gold in Electron Microscopy
}

\author{
J. $\mathbf{T}_{n}$ Fourie
}

National Physical Research Laboratory, Council for Scientific and Industrial Research, Pretoria, South Africa

\begin{abstract}
The techniques of transmission and scanning electron microscopy bave found unique application in the study of the microstructure of surfaces. In this application, the use of gold and its alloys has proved indispensable in producing images of the required contrast and detail. A brief survey is given here of the origin of contrast in these techniques and of how gold can be used to improve the quality of electron microscope images.
\end{abstract}

The development of electron microscopy during the first two of its five decades of existence was linked very closely to the availability of heavy metals such as gold. Following on the postulate of de Broglie in 1924 concerning the dual particle/wave nature of the electron and on the work of Busch (1) who demonstrated that axially symmetrical magnetic fields would focus electron beams, the first electron microscope was constructed in 1932 by Knoll and Ruska (2). However, in applying this new instrument it was quickly realized that samples prepared similarly to those for light microscopy did not produce satisfactory images. Principally, the contrast was too low for utilizing the high resolution of which the new microscopes were capable, since the smallest object detail $d$ that can be imaged in a specimen is inversely proportional to the contrast $C$, where $C$ is defined as the difference in intensity between two adjacent image points divided by their average intensity (3).

Specimens for the transmission electron microscopes which dominated the scene from 1932 to 1965 had to be extremely thin (less than $200 \mathrm{~nm}$ ). For this reason, suitable contrast could be obtained only by using heavy metals to increase artificially the contrast of surface detail in surface replicas by so-called 'shadowing', or of internal detail in biological sections by heavy metal staining techniques.

The use of gold and its alloys in electron microscopy is largely related to studies of the surfaces of solids. Such studies may be carried out indirectly by replication of the surface and subsequent transmission electron microscopy (TEM). Alternatively, an image of the surface topography can be obtained by scanning electron microscopy (SEM). In both instances, the evaporation of gold or of a gold alloy during the preparation stage is essential. At the present time, the majority of surface topogtaphy studies are being done by SEM rather than by TEM. This can be attributed to the relative ease with which SEM can be used for this purpose. It is not always realized, however, that the optımum resolution obtainable in the surface scanning mode of bulk specimens is usually about $30 \mathrm{~nm}$, whereas the replica/TEM technique consistently achieves surface resolutions below $3 \mathrm{~nm}$. Nevertheless, the emphasis for surface imaging is strongly on SEM and for this reason a meaningful estimate concerning gold consumption by electron microscopy units need only consider SEM installations.

The total annual amount of gold consumed world-wide in preparing electron microscope specimens can be estimated quite readily.
The number of active electron microscope groups in the world is probably about two thousand. On average, each group can be expected to perform three gold evaporation processes daily for its microscopes, that is $3 \times 250$ evaporations annually per group. Since a gold layer of approximately $10 \mathrm{~nm}$ is required on samples placed some $100 \mathrm{~mm}$ away from the source of evaporation, it can be calculated that a volume of about $1.2 \times 10^{-3} \mathrm{~cm}^{3}$ is used in each evaporation. Hence, a single group will consume around $0.9 \mathrm{~cm}^{3}$ or $17 \mathrm{~g}$ of gold annually. For two thousand groups the total consumption therefore amounts to about $34000 \mathrm{~g}$ of gold. The equivalent in troy ounces is 1.100 , which at a gold price of U.S. $\$ 500$ per ounce represents an annual expenditure of $\$ 550000$.

In this article the methods used for gold evaporation and sputtering in electron microscopy applications will be described, while the physical basis of contrast in TEM samples, such as replicas, and of surface topography of bulk samples in SEM will be considered, together with some illustrative examples.

\section{Replicas of Surfaces}

When very fine detail of surface topography is required, a replication technique yields the best results. Two-stage replica methods are usually preferred, because the sample is not damaged during replication. The essentials of the two-stage method are illustrated in Figure 1 and can be described as follows:

(1) The sample surface is flooded with a dilute solution of a plastic in a volatile organic solvent such as chloroform. After drying for 1 minute, the plastic can be stripped from the sample

(2) The plastic replica is placed in an evaporation plant and shadowed at an angle of $30^{\circ}$ with gold-palladium alloy, as will be described later. Thereafter, carbon is evaporated by resistance heating of pointed graphite electrodes, this time at normal incidence

(3) The plastic is dissolved, leaving behind a double layer of carbon and gold-palladium. Variations in the thickness of the latter reflect surface topography.

\section{Evaporation Methods for Gold Coating of Surfaces}

The method used for gold or gold-palladium alloy evaporation can be described with reference to Figures 2 and 3. For TEM samples such as replicas, a tungsten spiral (W in Figure 2) could be 
(a)

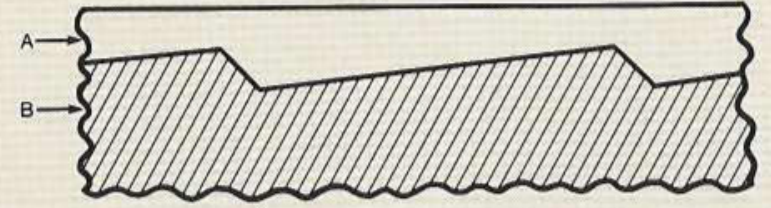

(b)

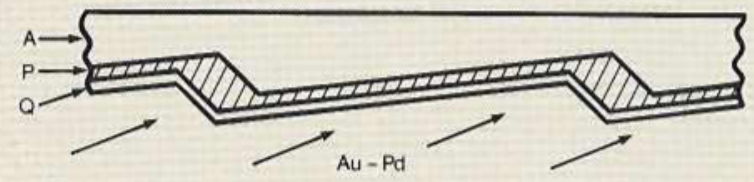

$\uparrow$

(c)

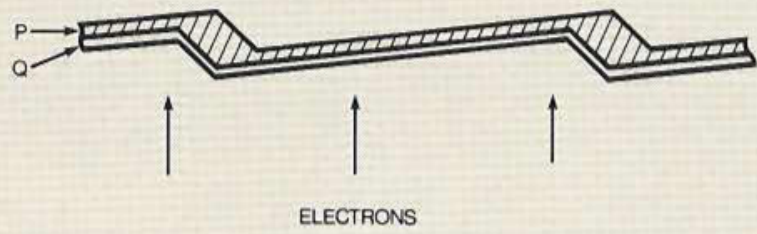

Fig. 1 Preparation of a surface replica for TEM

(a) The surface of the sample B is covered with a thin plastic layer A

(b) The plastic layer A is stripped from the sample and then 'shadowed' obliquely with gold-palladium alloy $\mathrm{P}$, followed by a vertical evaporation of carbon to form the thin carbon supporting layer $Q$

(c) The plastic layer is dissolved and the composite layer (P-Q) is transferred to the electron microscope for examination and photographing at high magnification

used to generate metal or alloy vapour. However, the planetary motion device $\mathrm{P}$ would then be removed and replaced by a stationary horizontal plate, the height of which relative to $W$ would be adjusted so that the angle subtended by W at the sample would be about $30^{\circ}$ when the latter is placed on the plate at a distance of $100 \mathrm{~mm}$ from W. The alloy $60 \mathrm{gold} / 40$ palladium weight per cent is available in the form of $0.2 \mathrm{~mm}$ diameter wire for evaporation onto replicas. The thickness of the goldpalladium deposit on the horizontal planes at the surface of the sample would be about $2.5 \mathrm{~nm}$ under such conditions, after a 10 mm length of gold-palladium wire had been evaporated.

For SEM, it is of paramount importance that the gold coating should be uniform in thickness, in order not to mask any of the surface features of the specimens. Therefore, for a non-planar surface, it is desirable that the gold atoms emanating from the gold source W should strike the specimen from all directions. This condition can be approximated by spinning the samples around their own axes and simultaneously spinning the table $P$, on which they are mounted, around its axis during the evaporation. This socalled planetary motion can be achieved by means of equipment as shown in Figure 2.

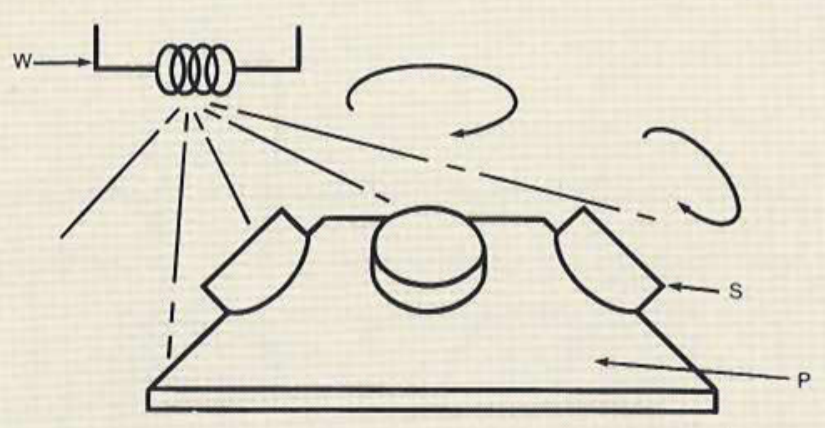

Fig. 2 Preparation of samples for SEM. The sample $S$ undergoes planetary motion during evaporation of gold wire for the tungsten spiral W.

Another very convenient method for achieving the multiangular incidence of gold atoms is sputtering, and an apparatus for this is shown in cross-section in Figure 3. Here, the gold atoms are sputtered from a ring-shaped target. When a high potential difference of between 1 and $3 \mathrm{kV}$ is established between electrodes within a rarefied atmosphere, an electrical discharge passes between them. The cathode electrode is then bombarded by positive ions of a residual gas such as argon. This bombardment results in atoms of the cathode material (gold) being sputtered off and transferred to nearby surfaces, upon which the atoms impinge from statistically random directions. Thus, excellent coverage is obtained, since the atoms penetrate even the reentrant cavities on very rough surfaces.

\section{Contrast in TEM}

The shadowed replica in Figure 1c may be considered as a general example for an elementary discussion of contrast in TEM. The main principle of high resolution replicas is that the layer of shadowed material should be thin and that there should have been very little surface diffusion of the sublimated heavy metal atoms after their impingement on the surface. Such replicas, when properly shadowed, can provide a surface resolution of 2 nm. (An electron mictoscope image with a resolution of $2 \mathrm{~nm}$ may be regarded as a medium resolution image since high resolution images in modern microscopes show detail of $0.2 \mathrm{~nm}$.) In medium resolution images, the contrast mechanism is related to the angle through which electrons are scattered as they pass through the specimen. As a general principle, the thicker the specimen and the higher the atomic number of the specimen material, the more electrons are scattered through a given angle away from the axial direction of the primary electron beam. The objective lens aperture in electron microscopes is positioned on the side of the specimen opposite to that on which the electrons are incident. Therefore, if electrons are scattered through an angle greater than the angle subtended by the objective aperture at the 


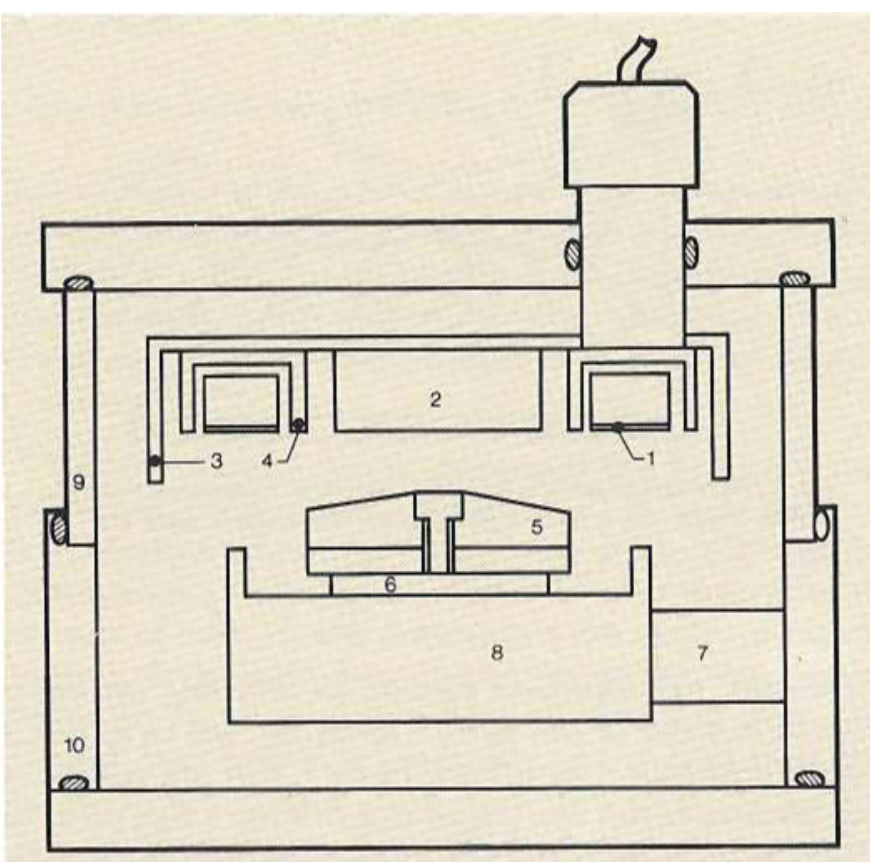

Fig. 3 Cross-section of a sputter coater for SEM sample preparationy 1 Gold target

2 Magnet

3 Iron pole piece

4 Aluminium shield

5 Specimen holder

6 Cooling module

7 Aluminium bar

8 Aluminium block

9 Glass jar

10 Vacuum collar

After (4)

specimen, they are intercepted by the aperture diaphragm and do not contribute to the image. Consequently, with reference to Figure $1 c$, it follows that the regions where the deposited goldpalladium alloy is thickest appear darkest and vice-versa. A good example of this is shown in Figure 4, which is the image of a goldpalladium/carbon replica of the surface of a plastically deformed single crystal of 97.5 copper $/ 2.5$ aluminium weight per cent alloy. The so-called slip steps on the surface show up as sharp dark lines, since the surfaces exposed by the 'slip' of (111) lattice planes when the crystal is deformed in tension make a large angle with the shadowing direction (as do the strongly inclined regions in Figure $1 \mathrm{~b}$ ).

The shadowing technique is also very useful in showing up surface protruberances or small particles composed of low atomic number elements, which normally would produce very little contrast. A good example of this is shown in Figures $5 \mathrm{a}$ and $5 \mathrm{~b}$ where so-called hydrocarbon contamination cones that are produced on thin foils when a narrow electron beam of $3 \mathrm{~nm}$ is

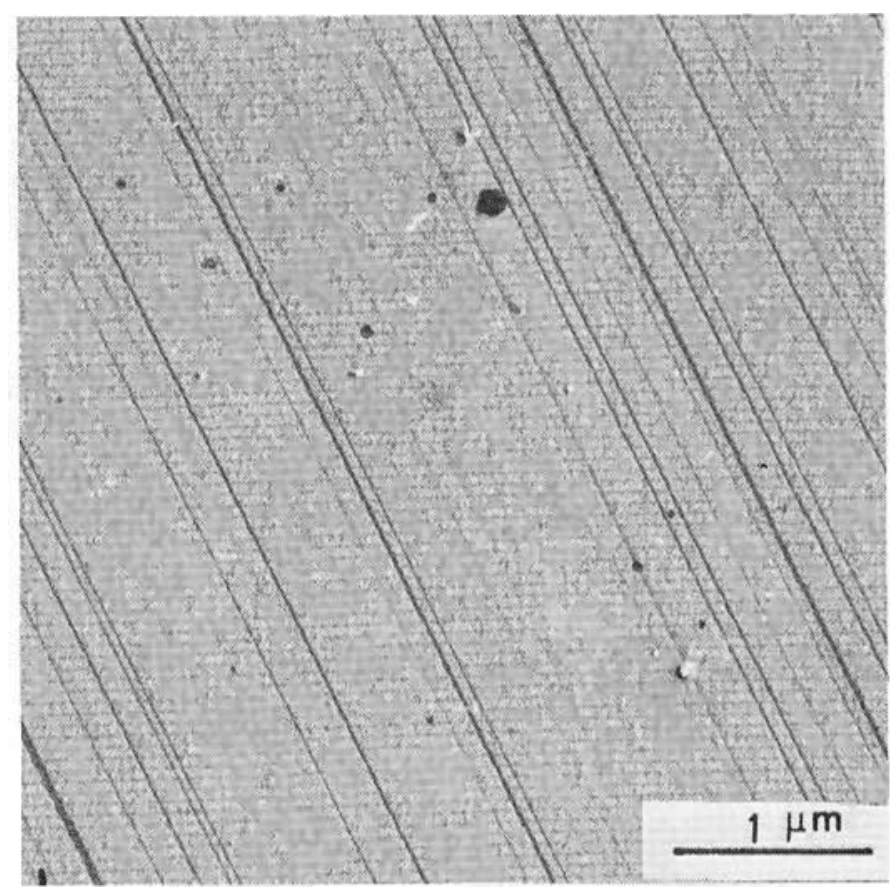

Fig. 4 TEM imaging of a replica of slip lines on the surface of a plastically deformed copper-aluminium alloy single crystal. The replica has been shadowed with gold-palladium alloy

allowed to impinge in one position for 2 minutes are visible. In Figure $5 \mathrm{~b}$ the cones on the shadowed side of the foil can be seen with much greater clarity than in Figure 5a, which is its unshadowed equivalent. It is evident from Figure $5 \mathrm{~b}$ that the goldpalladium alloy does not form a continuous layer. Instead, the metal atoms, upon arrival at the surface, tend to cluster into small 'islands' with a resulting 'grainy' appearance. The size of these islands affects the resolution from the replica. Obviously, the smaller the islands, the higher the obtainable resolution and, for this reason, gold-palladium alloy is preferred to pure gold for shadowing replicas, since the islands in an evaporated gold film tend to be considerably larger. However, in SEM, in which resolutions of less than $20 \mathrm{~nm}$ are tarely obtained in practice and in which 'graininess' is therefore not so important, pure gold is usually preferred, because of its excellent secondary electron coefficient and ease of evaporation.

\section{Contrast in SEM}

When high energy ( 10 to $50 \mathrm{kV}$ ) electrons impinge on the surface of a bulk specimen, several types of secondary radiation are excited while, in addition, some of the primary high energy electrons are backscattered. The present discussion, however, will be limited to image formation by secondary electrons only.

The contrast in a secondary electron image of an undulating surface can be understood on the basis of Figure 6. It can be shown (4) 

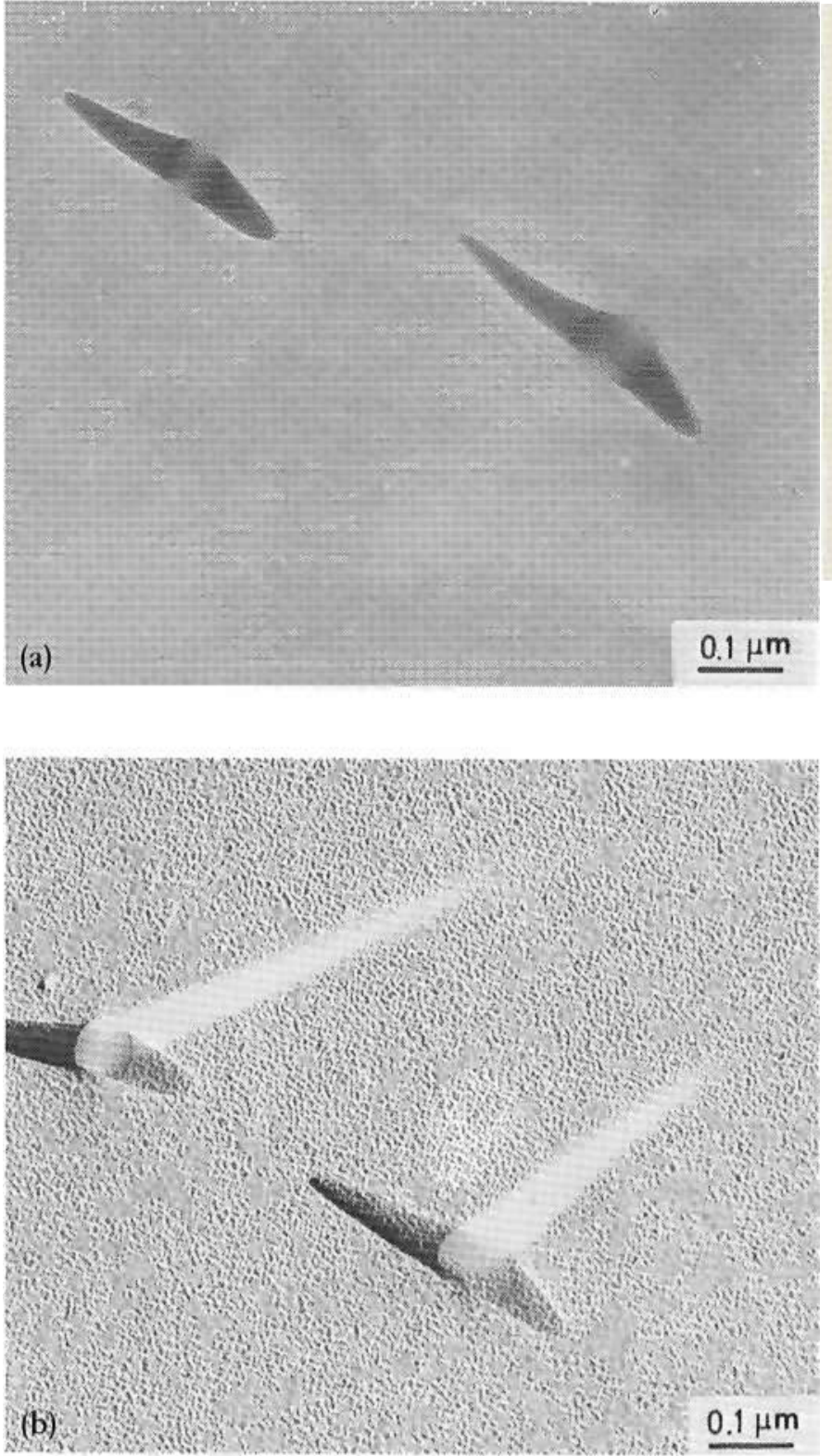

that surface elements which are at an angle to the horizontal will appear brighter than the horizontal surface elements on which the primary beam impinges at normal incidence. This contrast relation is described mathematically by the expression

$$
\mathrm{N}(i)=\mathrm{N}(0) / \cos i
$$

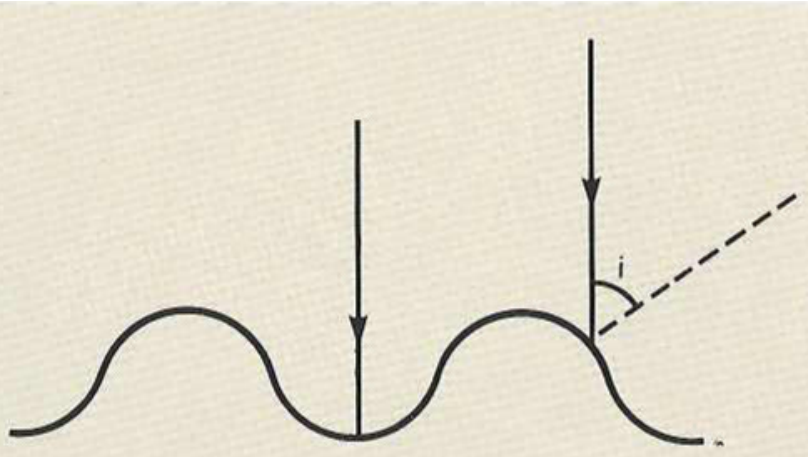

Fig. 6 The emission of secondary electrons from an inclined surface is governed by a cosine law, in which $i$ is the angle between the incident primary electron beam and the surface element normal

where

$i$ is the angle between the incident primary beam and the surface element normal

$\mathrm{N}(i)$ is the number of secondary electrons emitted per unit time from that surface element

$N(0)$ is the number of secondary electrons emitted per unit time for normal incidence of the primary beam on an equivalent surface element.

It is thus possible to interpret the images on this basis. An additional contrast effect follows from the fact that the detector is placed to one side of the specimen, away from the path of the incident beam. This leads to a 'perspective' type of image, and micrographs have a three-dimensional quality as is clearly shown by the image of a gold-coated tick in Figure 7.

The ultimate resolution in a scanning electron microscope is determined by the diameter of the electron beam which scans the specimen. In a thin foil specimen, and using the microscope in a scanning transmission mode, a resolution approaching the beam diameter can be attained fairly easily. However, in surface scanning of bulk samples, where secondary electrons produce the imaging signal, such resolutions are rarely possible. This is because the secondary electrons emanate from an area greater than that covered by the incident beam at a given instant. For this reason, resolutions better than $10 \mathrm{~nm}$ are not feasible by SEM. Moreover, it is important when working at high magnifications (about 50000 diameters) that the beam diameter should be about $10 \mathrm{~nm}$, this being determined by a number of factors. First, it follows from basic electron optics that the minimum beam diameter is related to the total current in the primary beam, which may not exceed a certain specified value for a certain minimum beam diameter. Therefore, the lower the beam current, the smaller the attainable beam diameter. On the other hand, the magnitude of the secondary electron signal is proportional to the primary beam current and thus, to obtain a favourable secondary 


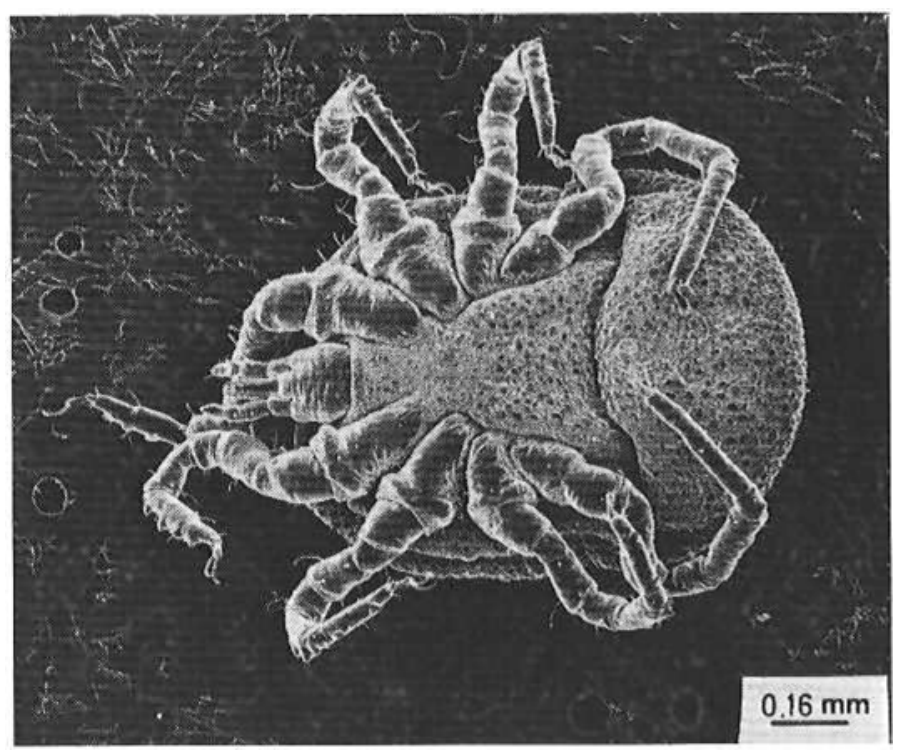

Fig. 7 SEM image of a gold-coated tick

signal-to-noise ratio, the primary beam current may not be lowered indiscriminately. The use of gold for coating a sample improves the attainable tesolution, because the metal has a very high secondary electron coefficient $\sigma$ where $\sigma$ is the number of secondary electrons emitted per incident primary electron. It follows that for a gold-coated sample, the current in the primary beam, and therefore also the beam diameter, may be considerably lowered relative to that used for an uncoated sample, without detriment to the intensity of the secondary electron signal. A 10 $\mathrm{nm}$ gold layer is sufficiently thin not to obscure surface detail while producing all of the aforementioned effects, thereby greatly improving the image of surface topography. An excellent example of the benefits provided by a gold coating is shown in Figures $8 \mathrm{a}$ and $8 \mathrm{~b}$. In Figure $8 \mathrm{a}$, a pearlitic steel structure is imaged without having been coated with gold, whereas in Figure $8 \mathrm{~b}$ the identical area is shown after coating with gold. The considerable enhancement of surface detail is evident.

\section{Summary}

A brief survey of the use of gold in transmission and scanning electron microscopy is presented. It is shown that in the imaging of surfaces for such mictoscopy, the use of gold or of its alloys in specimen preparation is of paramount importance and has contributed immeasurably to the improvement of both techniques, as well as to advances in the understanding of solid state processes investigated by them.

\section{Acknowledgements}

The author is indebted to Dr. N. R. Comins and Mrs. J. Harris for assistance and discussions.
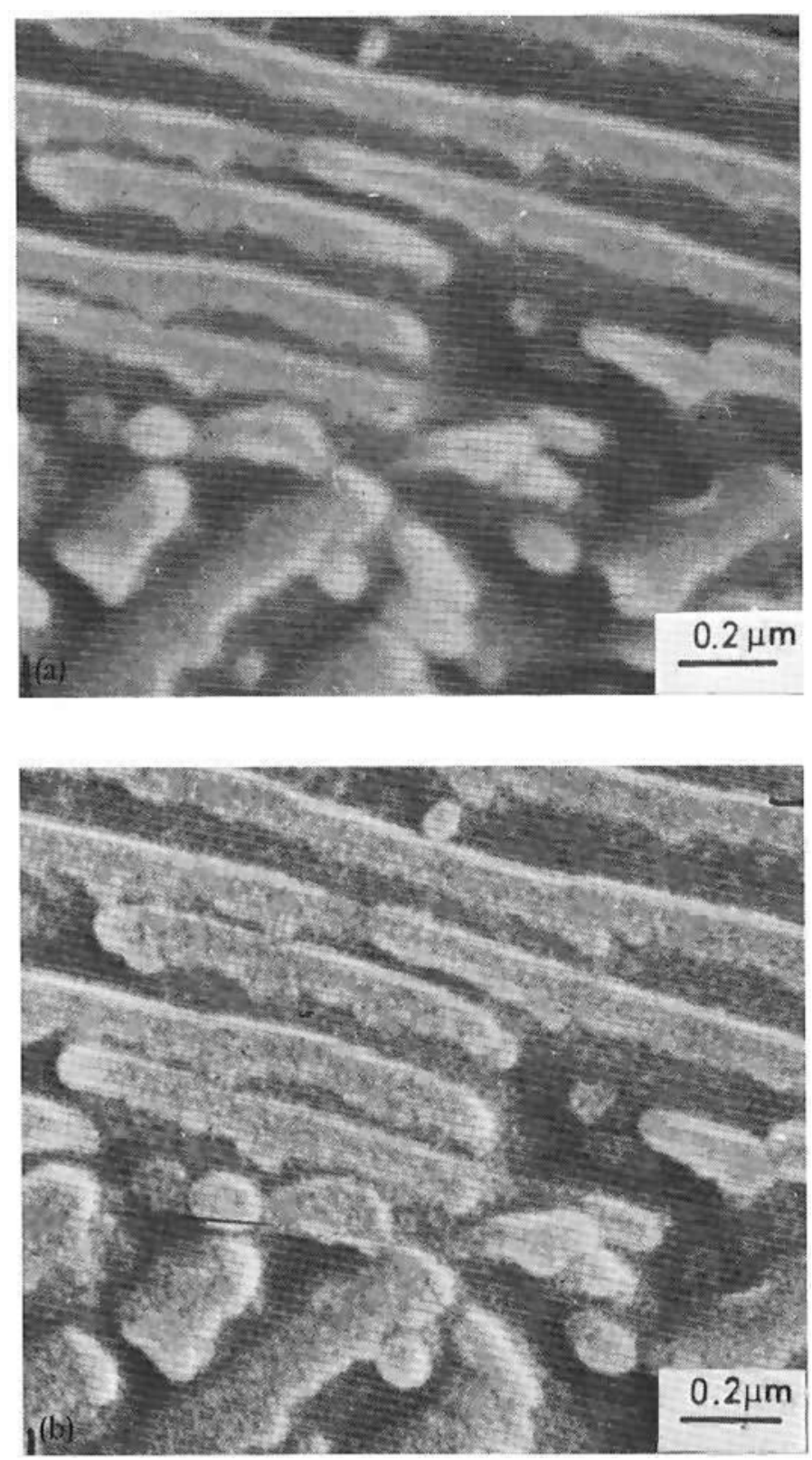

Fig. 8 High magnification SEM images of pearlite in a steel

(a) Uncoated steel sample

(b) Same area, but coated with about $5 \mathrm{~nm}$ of gold

References

1 H. Busch, Ann. Phys, 1926, 81, 974-993

2 M. Knoll and E. Ruska, Z. Phys., 1932, 78, 318-339

3 R. M. Glaeser, in 'High Voltage Electron Microscopy', edited by P.R. Swann, Academic Press, London, 1974, pp. 370-378

4 P. Echlin, 'Scanning Electron Microscopy' IITRI, 1977/1, pp. 463-470

5 P. J. Grundy and G.A. Jones, 'Electron Microscopy in the Study of Materials', Edward Arnold, London, 1976

6 J. T. Fourie, in 'Scanning Electron Microscopy/1981/1', SEM Inc., AMF O'Hare, IL. in press 